\title{
High Dynamic Range Imaging in Design Education
}

\author{
Ryan Southall, Senior Research Fellow \\ School of Architecture \& Design, University of Brighton, UK
}

\begin{abstract}
High Dynamic Range (HDR) imaging is a popular photographic technique that accurately captures the lighting conditions of the scene from which they are created. HDR images can subsequently be used to illuminate 3D virtual scenes realistically, or be a source for numerical lighting simulations. This paper discusses two workflows that have been developed using inexpensive digital cameras and open source software, supporting the interactive exploration of realistic lighting conditions, both qualitatively and quantitatively, in a digital environment. The paper first details the suitability of the qualitative workflow as a vehicle for teaching the technical aspects of lighting to architecture students, due to its relative ease of implementation, close relationship to existing design tools, and application to visual design work. Finally, the extension of this technique to enable simplified numerical analysis is discussed.
\end{abstract}

Keywords: Lighting, Rendering, Simulation 


\section{Introduction}

High Dynamic Range (HDR) imaging (Reinhard, et al., 2010) is a digital photographic technique that composites together a number of differently exposed conventional digital images of a scene to produce an image with a higher and more accurate brightness range. This enhanced brightness range is numerically coded into the image format, two of the most common of which are Radiance RGBe (.pic/.hdr extension) and OpenEXR (.exr extension).

The digital workflow for this photographic technique was first revealed in 1997 (Debevec, 1998) and was intended for the generation of realistic lighting sources for virtual environments. Subsequently, it forms a direct relationship between the physical site and digital design, and as such provides a viable tool for the teaching of the technical aspects of light within an architectural context.

\section{Educational context}

The use of HDR imaging in this architectural education context has sprung from the desire to match core technological teaching more closely with studio design work.

The optimal approach to the teaching of design and technology in an architectural education context has been often debated (Watson, 1997), and perceived to be problematic (Austin, 2007; Fontein, 1997), but the need has never been greater for architects and other built environment professionals to understand the relationships between the environment and the buildings they construct (Jenks and Dempsey, 2005). If environment and design are taught in isolation then reliance is placed upon students to find their own discourse between the two in order to satisfy both these elements of their curriculum. If the two are integrated then a more 'complete' design education can be delivered (Watson, 1997), but the issue then becomes how to make environment and design relevant to the each other throughout the learning process.

A number of approaches have been used for the teaching of lighting in architectural environments, and its importance is clear from the number of lighting specific analysis aids that have been designed for architectural use. These include: basic rules of thumb, the LT method, Daylighting nomographs (Moore, 1986), BRS protractor (Moore, 1986), Graphical Data Display Manager (GDDM) (Millet and Bedrick, 1980), Leso-Dial (Paule and Scartezzini, 1997), Radiance (Larson and Shakespeare, 2004) and LightScape.

One approach used staged physical and computational exercises (Fontein, 1997) which have been successful in terms of 'hands-on' experimentation, but less successful in the latter stages at the application of lighting to architectural form. Another (Millet and Loveland, 1997) used model making as a vehicle for, firstly, precedent study, followed by a student's own design, with a final calculation assignment based on studio work. A third approach (Egan and Olgyay, 2002) also used scale physical models to allow 
students to successfully test and develop daylighting design systems. An extensive programme of lighting education at the University of Washington based upon physical experimentation (Millet and Loveland, 1997) is another. Yet another approach moved from a theoretical to a progressively complex analytical approach (Dubois, 2006). It is clear from these studies that enabling students to undertake 'hands-on' experimentation and iteration is one successful approach to the teaching of lighting.

The use of HDR imaging presented here attempts to maintain this success by enabling easy experimentation and play whilst teaching light in a manner directly related to the actual site in which an architectural intervention is proposed. This directly addresses both technology and design by introducing a technological process (light) that directly improves final digital visual output. The importance of the role light can play in this context has long been recognised (Millet and Loveland, 1997), and its influence on the entire design process, incorporating both the aesthetic and the technical, has been noted. These technical and aesthetic qualities make it an ideal vehicle to increase understanding between, and integration of, design and technology in architectural education.

Although the physical approaches mentioned have an invaluable tactility there are some advantages that modern computational techniques can bring to bear on the subject. Faster design iterations, full scale models, almost limitless material and form flexibility and, with HDR images generated at the proposed site, a direct connection to the environment of the students' buildings, are all more easily achieved in the computational realm.

Students can, for example, follow the lighting data they themselves have generated from acquisition at their physical site, through analysis and design iteration, to final presentation. This has a number of advantages in this context:

- The lighting data they gather are specific to their site, weather and the time of day that the original photos were taken. This strengthens the connection between real context and subsequent design work.

- Initial acquisition of the photographic shots is easily integrated into existing physical work (e.g. site survey).

- These physically generated data can easily be integrated into existing digital workflows.

- Once embedded within the digital workflow, optimisation of design in terms of materiality and form can be achieved from the perspective of real lighting conditions. Lighting analysis in architectural education is too often a form of evaluation rather than as a constituent element of the design process. This feature of the use of HDR imaging serves to facilitate this critically important iterative integration within design. 
- The integration of these quite technical data improves the visual quality of the final work, which helps maintain student engagement in an arts orientated discipline.

It has been argued (Pittman, 2005) that computing has merged so integrally with design studio that its co-existence with design has become seamless. Its use, however, has predominantly been only to compose, manipulate and present form, and this research argues that the ability to assess function and site-specific performance is required if computational capability is to be optimised in design and design critique.

\section{Generating HDR Images}

\section{Introduction}

The two most common techniques for the generation of HDR panoramic images, mirror ball and panoramic (Bloch, 2007), were assessed for ease of use and quality of final image. Although the mirror ball technique has some advantages in terms of ease of use as fewer shots are required, the relative complexity of software post-processing and difficulty in achieving good quality images makes the panoramic technique more suitable for this context. To reduce the relatively high number of shots required to create a $360^{\circ}$ panorama this way a fish-eye lens has been used. This limits the number of distinct sets of shots required to four. The immediate ground in the scene is missing (Figure 1), but for most lighting scenarios where the majority of lighting comes from the sky, or more distant ground, this has been considered an acceptable trade-off for simplicity.

An additional consideration for the completion of the HDR generation workflow is the software required to composite the images taken into one HDR panorama. Fortunately the free, open-source and multiplatform photo stitcher Hugin can natively composite and stitch bracketed shots into complete $360^{\circ} \mathrm{HDR}$ panoramas.

\section{Final set-up and workflow}

After investigation into different hardware and software options the following set-up was chosen:

Camera - Samsung gx-20 (up to 5 bracketed shots in 2EV steps)

Lens - Samyang 8mm, aspherical, fish-eye lens

Software - Hugin, open source, free and multiplatform panorama stitcher.

A detailed breakdown of the process of creating HDR panoramas, and the hardware and software considerations for doing so, can be found at the HIDE project website (Southall, 2011).

The overall cost of the system was approximately $€ 900$, which although significant, should not be prohibitive for most educational institutions. 


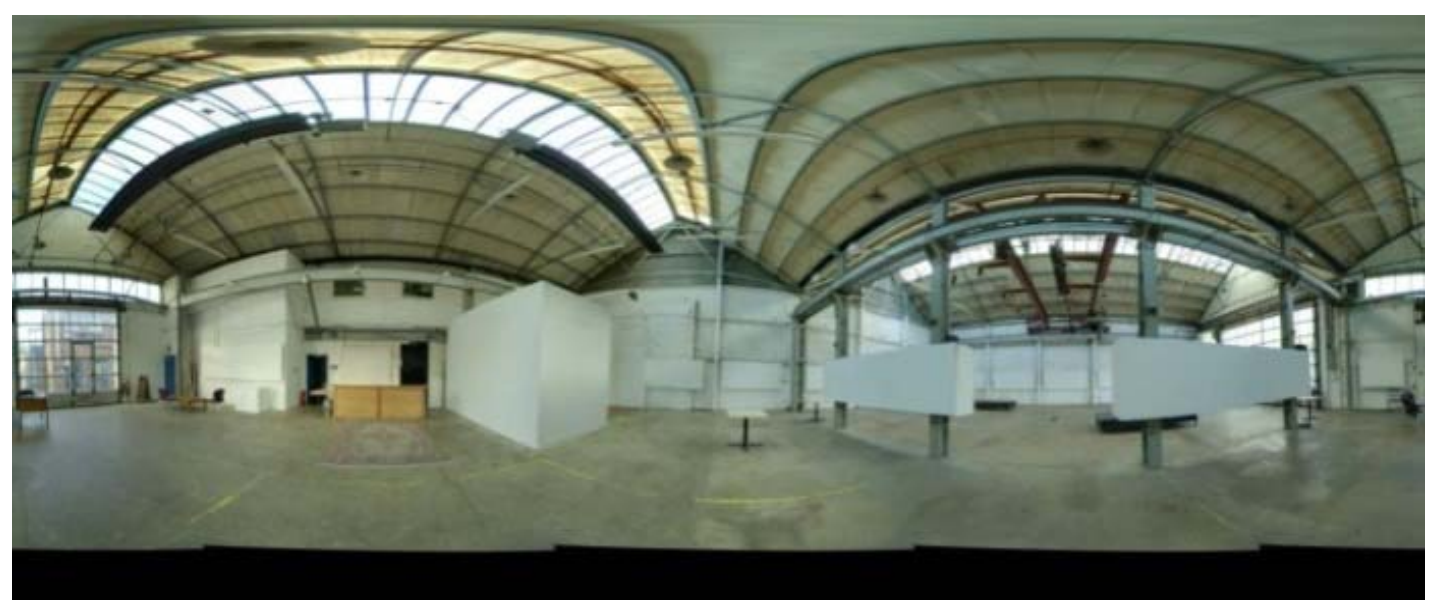

Figure 1 Final tone mapped HDR panorama of an interior scene.

\section{Using HDR Images}

An HDR light probe image represents the brightness and directional lighting environment for the position and time the original photographic shots were taken. When then applied as an environmental lighting source around a virtual 3D environment, any object within that environment is illuminated as if it was situated in the same time and place as the camera. All details of the lighting environment are captured, so the subtleties resulting from overshadowing, reflection from surrounding objects etc. is also maintained within the image. The application to architectural site surveying is clear.

Once an HDR image is in a common light probe geometric format, like angular map or lat-long, their integration into digital design workflow is easily accomplished. Most renderers, either stand-alone or integrated into 3D modelling packages, can accept the light probe as an automatic scene light source. Usually it is no more involved than picking the requisite file from a dialogue and possibly orientating it to match the orientation of the 3D model. At the University of Brighton's School of Architecture \& Design, Cinema4D and Vectorworks are extensively used, and both accept HDR panoramas as an environmental light source. This is a key advantage of HDR imaging in a design education environment, especially architecture, as students will be familiar with these packages from 3D design elements of their course, and the integration of this accurate lighting data with their design work is thus streamlined. As long as the renderer used is of reasonable accuracy, rendered objects will look as they would at the physical scene. This is especially the case if using modern 'un-biased' rendering systems like Maxwell (http://www.maxwellrender.com ) or LuxRender (http://www.luxrender.net ) (Figure 2). 


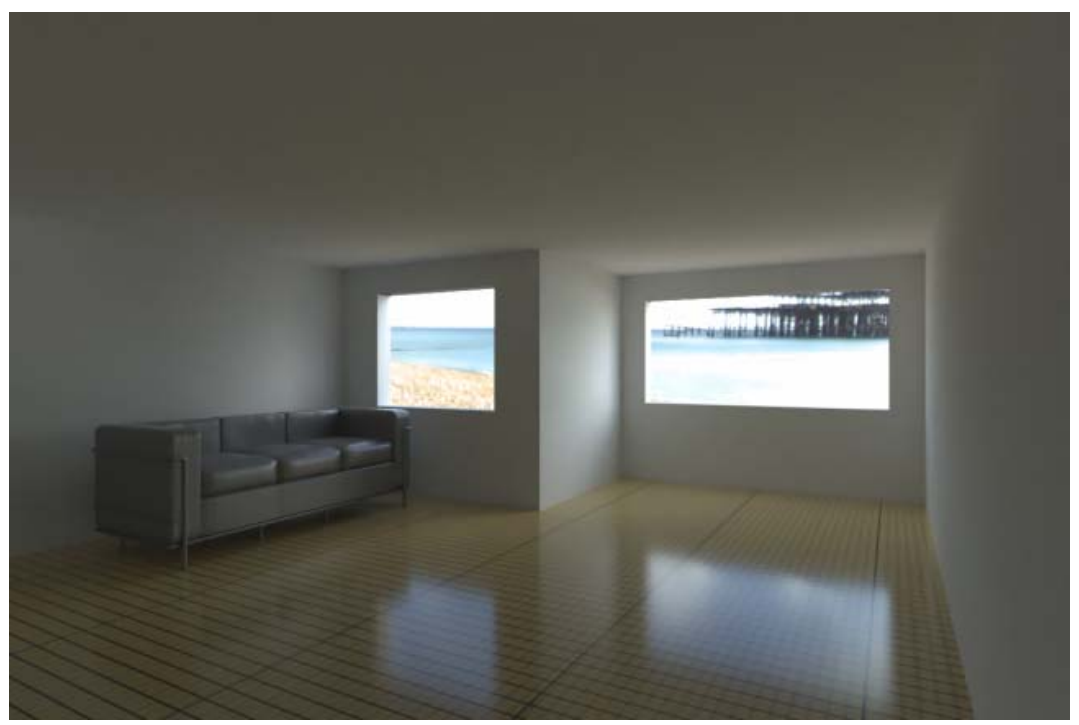

Figure 2 LuxRender rendering with HDR sky background

One of the advantages of the panorama technique of HDR generation is the high quality of the final image. This allows the generated light probe to be not only the light source for a virtual scene but also the background, placing the student's virtual design very much in its correct geographical context.

\section{Quantitative Analysis}

This initial work has centred on the qualitative analysis of site specific HDR lighting conditions, but quantitative analysis is also possible and the resultant site specifity can result in greater numerical accuracy than conventional quantitative analysis (Inanici, 2009). Achieving quantitative analysis with HDR lighting sources not only requires numerically validated HDR (Inanici, 2006) source files, but a numerical analysis method that can take full advantage of the subtleties resident in the lighting source.

A central ethos of this project is the use of low-cost hardware that requires no great institutional investment, and enables students' experimentation and play. This presents some challenges in terms of achieving numerical accuracy. The camera used in this project has the largest EV range in the price bracket desired that could be found, but a range of $8 \mathrm{EV}$ is still not enough to capture the whole dynamic range of a sunlight scene, which would require 17EV (Stumpfel et al., 2004). Calibration of the camera within the capable dynamic range would however generate accurate lighting sources for non clear sky scenarios. To enable quantitative analysis under clear sky conditions an alternative approach has been used whereby HDR panoramas are automatically generated based on CIE standard mathematical sky descriptors.

Assuming an acceptable level of physical accuracy has been achieved, HDR images can also be used as input to numerical lighting simulation packages, such as Radiance (Larson and Shakespeare, 2004), for the prediction of location specific lighting metrics. An issue with Radiance is its relative complexity of use, especially within the context of 
a design orientated learning environment (Compagnon, 1997; Mansy, 2004). To maintain simplicity throughout the workflow a set of scripts, called the Lighting Visualiser (LiVi) and available from the HIDE website (Southall, 2011), have been written to interface the open-source modelling tool Blender with the lighting simulation programme Radiance. The student, or CIE, generated HDR panoramic skies can be specified as a lighting source within Blender, the scene exported to Radiance for simulation and the results brought back into Blender for visualisation. Any geometry within the 3D model, like a floor (Figure 2) can be specified as calculation points for Radiance, which will then return values for the chosen lighting metric that can colour the specified Blender geometry. As the scripts hide a lot of the complexity of using Radiance, students have access to a complete simplified workflow from lighting data acquisition, through analysis, simulation and design iteration to final visual presentation, both photo-real and numerical.

Blender offers a number of advantages for the visualisation of numerical Radiance data. Like Radiance it is free, open-source and cross-platform allowing students to install and use the software without licensing or compatibility issues.

Additionally, it is a polygon, as opposed to a solid modeller, meaning Blender geometry is composed of vertices, edges and faces as opposed to mathematical descriptions of form. This is advantageous here as Radiance, via its rtrace component, can calculate lighting metrics at particular Cartesian points within the 3D virtual scene, and these points can be corresponding vertex or face co-ordinates of the Blender geometry. Generating sensor points within the model is therefore as simple as designating existing geometry with the requisite grid resolution. Another advantage is the ability to colour geometry at the vertex or face scale. This is an unusual ability in 3D modellers and results from the use of Blender to create game environments, with baked-in textures, using the inbuilt game engine.

Blender's use of the Python scripting language to control the Blender user-interface, model geometry and materiality, coupled with Python's excellent text processing and programme control capabilities, allows for the automatic control of Radiance and the creation of the LiVi user interface that presents a sub-set of Radiance parameters to the user. 


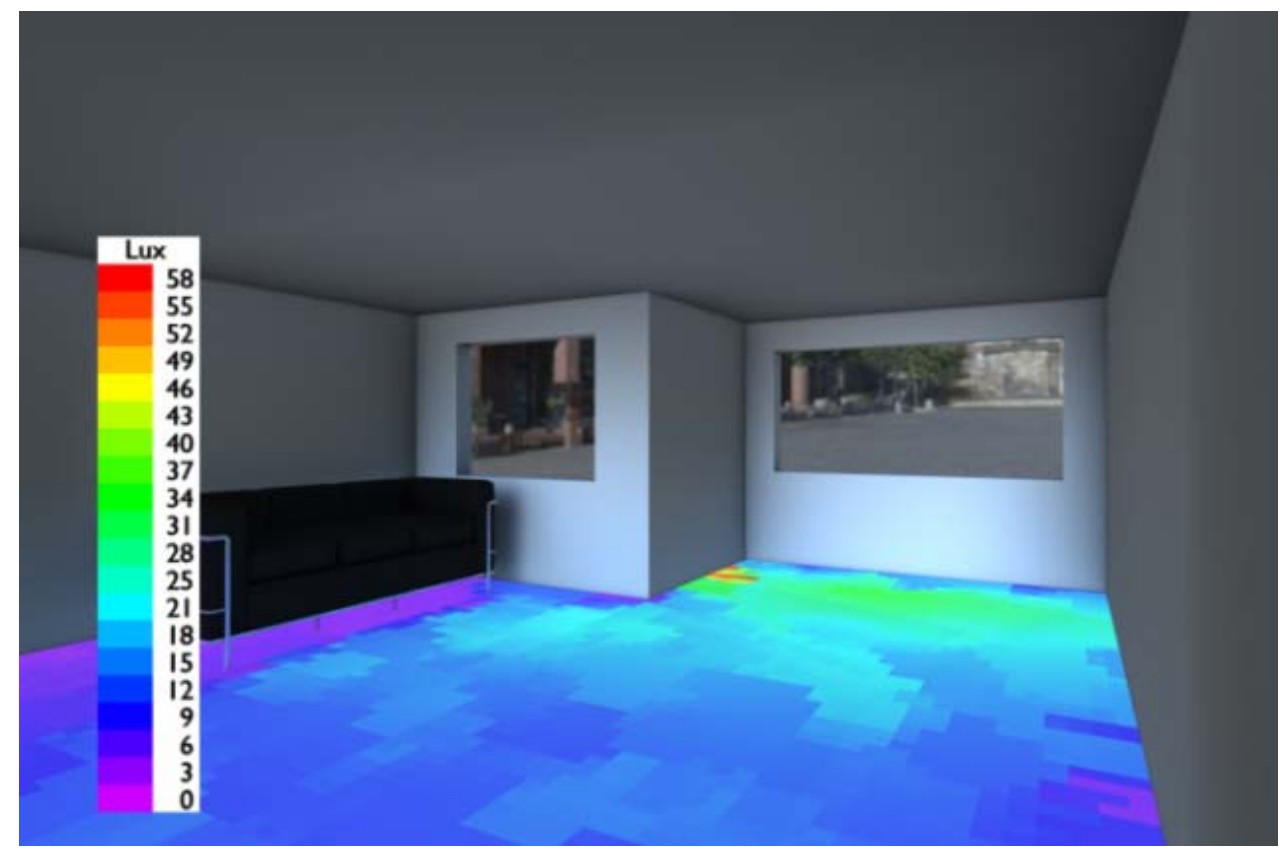

Figure 3 Blender rendering of Radiance results with an HDR sky background

Finally, and perhaps most importantly, Blender is a complete visualisation and rendering suite (although not a CAD environment). Traditional visualisation work can therefore be achieved within Blender and numerical lighting analysis can be quickly accomplished without having to learn a new software package. This encourages quick and iterative play, which has proven to be the most effective element of the physical teaching methods previously discussed.

\section{Teaching Methodologies}

Three distinct teaching methodologies were employed to integrate these developed HDR based qualitative and quantitative analyses into Brighton's architecture school, and wider arts faculty, teaching programmes. These three distinct methodologies consisted of:

- Presentation of hardware and software workflows for self-guided learning.

- Provision of resources and instruction within a specific, marked course structure.

- As an integral component of a technical unit of study.

The first method depended heavily on design studio context, as this is where most student work is carried out. This did however raise a number of issues. Site access and location is an important consideration in cases like these as students are being given the opportunity to borrow and use, what at least is perceived to be, expensive hardware equipment. Distant sites with limited access, or busy public spaces, are not as suitable in such contexts as close, controlled, easily accessible environments. Planning site location in a design studio to cater for one specific technical teaching 
technique would, however, be highly unusual. This technical teaching strategy therefore, like most others, will be more or less suitable for use in design studio based on contextual issues defined within design studio. For effective integration of technical processes within the design studio at all times, it is likely that a range of technical learning techniques need to be available that would be applicable to a range of building types, location etc. It is also important that any technical technique is closely associated with an aim in the design studio, and is seen as being at least one way to achieve that aim. If this is not the case then student motivation to engage with the process will be low.

The second method used a more traditional taught course structure to, stage-by-stage, go through the process of HDR photography, generation and use. The course took students from different schools within the faculty, but the majority were from architecture. Students were allowed to pick a site in and around Brighton that interested them from a lighting perspective. This removed issues regarding site access and safety.

The course dealt with many aspects of digital design including 3D form creation, animation and rendering, and as such was an excellent vehicle for teaching a complete digital workflow. The course utilised Hugin for the HDR panorama creation, and Blender for the HDR application, modelling, animation and rendering. Both tools are free, open-source and multi-platform, which allowed student to work away from the course on any aspect of the workflow. In addition Blender, especially, has a wealth of on-line resources describing its functionality that students could access.

All but two of the 12 students on the course managed to create and apply their own HDR panoramas as a lighting source to animated forms and materials they had created. Feedback from the course was positive and the animation element was found to be the most interesting. This interest in animation in an architectural education context is of note as moving images are rarely permitted as an assessable piece of work, and indicates that broadening the types of media that can be submitted could help engage some students, and keep abreast of rapid changes in digital design capabilities.

The third method involved the use of the LiVi scripts within Blender to allow students to use HDR images to conduct their own lighting analysis of their designs. The use of the LiVi scripts was attached to a technical unit of study and was presented as a way to complete the more advanced phase of the unit. Students were initially taught how to use the software, which they could download from the University's intranet service or use on Mac computers within the school, and then given weekly support with on-going usage issues. Ten students used the LiVi scripts to analyse and modify their 3D designs, roughly using the following process:

- Import 3D geometry into Blender from their current digital workflow 
- Check and reassign material definitions

- Specify calculation points

- Calculate and visualise the chosen lighting metric

- Make design changes based on the results

- Export the results back into their digital workflow for final presentation

The beginning and end of this process could be omitted if students were already using Blender for 3D composition and visualisation.

Although students were unfamiliar with lighting simulation most were able, even in the short time available, to analyse their current design and make informed design changes based on the results. Feedback was generally positive and students were surprised that they would have the opportunity to achieve accurate lighting simulation for themselves. Feedback from the students did highlight the lack of feedback from the LiVi scripts themselves, which made debugging difficult for an inexperienced user. This has now been improved in the latest version of the LiVi scripts.

\section{Conclusions}

The workflows presented deliver methods of relating the illumination of a relevant physical context or location with a qualitative and quantitative lighting analysis. Their strengths largely derive from this particular combination, which is difficult to achieve with other methods. To achieve this with purely computer based techniques would involve some effort to build an accurate digital representation of the surroundings with accurate lighting characteristics. Any attempt to imitate subtleties in sky colour would be even more difficult, and both of these activities would probably require some kind of on-site photographic survey work anyway. To achieve this connection with pure physical methods would either require the placing of a physical model at the physical site or within an accurate physical lighting delivery system. Accurate physical lighting systems that can characterise custom subtleties in ambient lighting environment are prohibitively expensive, and taking a physical model to a relevant site makes 'handson', iterative, refinement of the design hard to achieve.

The combinations presented here do, however, allow for the some of the subtleties of a real lighting situation to be expressed by the lighting source, whilst allowing quick design experimentation and play via the use of digital tools that are already part of general architectural education.

It is this 'hands-on', playful, element to lighting analysis and design that has proved to be the most successful component of the previous, more physical methodologies, discussed here. A weakness, however, remains close integration with design elements of the course. Of the three teaching methodologies employed the last has come closest to achieving a close integration of design and technology in an architectural context, 
and it also maintains the quick, iterative and playful component of previous approaches. The iterative component is a function of the simplicity of the LiVi interface, the subsequent ease of lighting analysis, and the integration of the results back into the 3D design software.

This paper has presented a number of approaches to the teaching of a technological component of architectural education, light, by the use of High Dynamic Range (HDR) imaging. Cost-effective and simplified workflows have been constructed to provide the easiest method to generate, use and integrate these images with student design work. When backed up with lectures, text and video based tutorials these techniques provide a powerful vehicle for the technical teaching of light, whilst at the same time improving the visual rendered quality, or numerical accuracy, of final design work.

\section{Acknowledgements}

The author would like to thank the Centre for Education in the Built Environment (CEBE) for funding this project. 


\section{References}

Austin, M. (2007) Integration of design and technology. Available at: http://epress.lib.uts.edu.au/dspace/bitstream/handle/2100/464/Austin Integration.pdf

Bloch, C. (2007) The HDRI Handbook: High dynamic range imaging for photographers and CG artists. RockyNook Santa Barbara.

Compagnon, R. (1997) The Radiance simulation software in the architecture teaching context. Paper presented at the $2^{\text {nd }}$ Florence International Conference for Teachers of Architecture, Firenze, Italy, 16-18 October 1997.

Debevec, P. E. (1998) Rendering synthetic objects into real scenes: Bridging traditional and image-based graphics with global illumination and high dynamic range photography. Proceedings of SIGGRAPH, Orlando, Florida, July 1998, pp.189-198.

Dubois, M. C. (2006) Integration of daylight quality in the design studio: From research to practice: Proceedings of PLEA 2006-The $23^{\text {rd }}$ Conference on Passive and Low Energy Architecture. Geneva, Switzerland, 6-8 September 2006.

Egan, M. D. \& Olgyay, V. W. (2002) Architectural lighting. New York: McGraw-Hill.

Fontein, L. (1997) Teaching lighting to architecture students: Technology as design inspiration: Proceedings of Right Light 4, Copenhagen, Denmark, pp.159-163.

Inanici, M. (2006) Evaluation of high dynamic range photography as a luminance data acquisition system. Lighting Research and Technology, 38 (2), 123-136.

Inanici, M. (2009) Applications of image based rendering in lighting simulation: Development and evaluation of image based sky models. Proceedings of the Building Simulation Eleventh International IBPSA Conference, 27-30 July 2009, Glasgow.

Jenks, M. \& Dempsey, N. (2005) Future forms and design for sustainable cities. Oxford: Architectural Press.

Larson, G. \& Shakespeare, R. (2004) Rendering with radiance: The art and science of lighting visualization. New York: Booksurge Llc.

Mansy, K. (2004) A user-friendly procedure to visualize the hourly quantitative performance of daylighting systems. Solar Energy, 77 (4), 373-380. Special Issue Millet, M. S. \& Bedrick, J. R. (1980) Graphic daylighting design method. Washington, DC.: Lawrence Berkeley Laboratory/U.S. Department of Energy.

Millet, M. \& Loveland, J. (1997) Teaching light: Its Importance in architectural education: Proceedings of Right Light 4, Copenhagen, Denmark, pp.169-173.

Moore, F. (1986) Concepts and practice of architectural daylighting. New York: Van Nostrand Reinhold. 
Paule, B. \& Scartezzini, J. (1997) Leso-dial, a new computer-based daylighting design tool. Proceedings of Right Light 4, Copenhagen, Denmark, pp.93-97.

Pittman, J. H. (2005) Computing in western architectural education. International Academic Seminar of Architecture Education. National Supervision Board of Architecture Education (China), (2005).

Reinhard, E., Heidrich, W., Pattanaik, S., Debevec, P., Ward, G., \& Myszkowski, K. (2010) High dynamic range imaging: Acquisition, display, and image-based lighting. Boston, MA: Morgan Kaufmann.

Southall, R. G. (2011) HIDE project website. Available at: http://arts.brighton.ac.uk/projects/hide

Stumpfel, J., Jones, A., Wenger, A., Tchou, C., Hawkins, T. \& Debevec, P. (2004) Direct HDR capture of the sun and sky. Proceedings of SIGGRAPH 2004.

Watson, D. (1997) Architecture, technology, and environment. Journal of Architectural Education, 51(2), 119-126. 